\title{
THEORETICAL ASPECTS OF SPIN PHYSICS *
}

\author{
DANIËL BOER \\ Department of Physics and Astronomy, Vrije Universiteit Amsterdam \\ De Boelelaan 1081, NL-1081 HV Amsterdam, The Netherlands
}

\begin{abstract}
A summary is given of how spin enters in collinearly factorizing processes. Next, theoretical aspects of polarization in processes beyond collinear factorization are discussed in more detail, with special focus on recent developments concerning the color gauge invariant definitions of transverse momentum dependent distribution and fragmentation functions, such as the Sivers and Collins effect functions. This has particular relevance for azimuthal single spin asymmetries, which currently receive much theoretical and experimental attention.
\end{abstract}

\section{Introduction}

The goal of QCD spin physics is to understand the spin structure of hadrons in terms of quark and gluon properties. For this purpose one studies polarization effects in high energy collisions, where one or more large energy scales may allow a factorized description. This means that cross sections factorize into quantities that describe the soft, nonperturbative physics and those that describe the short distance physics, which is calculable.

\section{Spin in collinearly factorizing processes}

The polarized structure functions $g_{1}$ and $g_{2}$ of Deep Inelastic Scattering (DIS) of polarized electrons off polarized protons (or other spin- $1 / 2$ hadrons), $\vec{e} \vec{p} \rightarrow e^{\prime} X$, appear in the parametrization of the hadronic part of the cross section, i.e., in the antisymmetric part of the hadron tensor

$$
W_{A}^{\mu \nu}=\frac{i \epsilon^{\mu \nu \rho \sigma} q_{\rho}}{P \cdot q}\left[S_{\sigma} g_{1}\left(x_{B}, Q^{2}\right)+\left(S_{\sigma}-\frac{S \cdot q}{P \cdot q} P_{\sigma}\right) g_{2}\left(x_{B}, Q^{2}\right)\right],
$$

with hadron momentum $P$ and spin vector $S$, photon momentum $q$, $x_{B}=Q^{2} / 2 P \cdot q$ and $Q^{2}=-q^{2}$. The definition of structure functions is independent of the constituents of the hadron. However, the operator product expansion or the PQCD improved parton model allows one to go to the quark-gluon level, such that the structure functions are expressed

*Talk presented at the Ringberg Workshop "New Trends in HERA Physics 2003", Ringberg Castle, Tegernsee, Germany, September 28 - October 3, 2003 
in terms of parton distribution functions (see Fig. 1). The two-quark

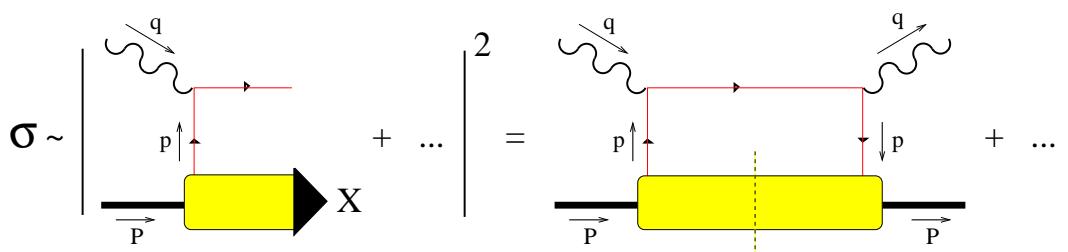

Figure 1. The $\gamma^{*} p$ cross section can be expanded in terms of parton correlators.

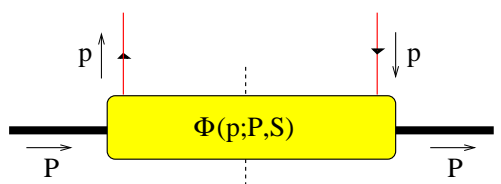

Figure 2. The two-quark correlation function $\Phi$, which depends on the hadron momentum $P$, quark momentum $p=x P$ and hadron spin vector $S$.

correlation function $\Phi(p ; P, S)$ (Fig. 2), or $\Phi(x)$ in short, is defined as

$$
\Phi(x)=\int \frac{d \lambda}{2 \pi} e^{i \lambda x}\langle P, S|\bar{\psi}(0) \mathcal{L}[0, \lambda] \psi(\lambda)| P, S\rangle,
$$

where the path-ordered exponential (also simply called 'link')

$$
\mathcal{L}[0, \lambda]=\mathcal{P} \exp \left(-i g \int_{0}^{\lambda} d \eta A^{+}\left(\eta n_{-}\right)\right),
$$

is not inserted in an ad hoc way to make $\Phi(x)$ color gauge invariant, but can actually be derived ${ }^{1}$ ( $n_{-}$in Eq. (3) is a lightlike direction). This $\Phi(x)$ is parametrized in terms of parton distribution functions. For longitudinal spin or helicity the (leading twist) parton distributions are $\Delta q, \Delta \bar{q}, \Delta g$ and for transverse spin they are $\delta q, \delta \bar{q}(\delta g=0$ due to helicity conservation):

$$
\begin{aligned}
\operatorname{Tr}\left[\Phi(x) \gamma^{+}\right] & \sim q(x), \\
\operatorname{Tr}\left[\Phi(x) \gamma^{+} \gamma_{5}\right] & \sim \lambda \Delta q(x), \\
\operatorname{Tr}\left[\Phi(x) \gamma_{T}^{i} \gamma^{+} \gamma_{5}\right] & \sim S_{T}^{i} \delta q(x) .
\end{aligned}
$$

From inclusive DIS, or more specifically, from the measurement of the structure function $g_{1}(x)$, one has obtained experimental information on $\Delta q(x)+\Delta \bar{q}(x)$, and implicitly on $\Delta g(x)$ via evolution. More information about $\Delta \bar{q}$ and $\Delta g$ will be obtained from polarized $p p$ collisions at 
RHIC (BNL) and from (semi-)inclusive DIS data of COMPASS (CERN), HERMES (DESY) and JLAB. In contrast, transversity $(\delta q)$ is completely unknown (no data). It cannot be measured in inclusive DIS, where it is heavily suppressed. The reason is that it must be probed together with another helicity flip. There are two types of collinearly factorizing processes that serve this purpose:

- Processes with two transversely polarized hadrons, e.g. $p^{\uparrow} p^{\uparrow} \rightarrow$ $\ell \bar{\ell} X, p^{\uparrow} p^{\uparrow} \rightarrow \operatorname{jet} X, e p^{\uparrow} \rightarrow \Lambda^{\uparrow} X$ or $p p^{\uparrow} \rightarrow \Lambda^{\uparrow} X$

- Processes sensitive to the two-hadron interference fragmentation functions 21345, such as e $p^{\uparrow}$ or $p p^{\uparrow} \rightarrow\left(\pi^{+} \pi^{-}\right) X$, where the angular distribution of final state hadron pairs is expected to be correlated with the transverse spin direction

This last option exploits the fact that the direction of produced hadrons can be correlated with the polarization of one or more particles in the collision. This is not merely a theoretical idea, but also has been seen in experiments, namely in single spin asymmetries in hadron and lepton pair production. Large single spin (left-right) asymmetries have been observed in $p p^{\uparrow} \rightarrow$ $\pi X \underline{678}$, where the pions prefer to go left or right of the plane spanned by the beam direction and the transverse spin, depending on whether the transverse spin is up or down and depending on the charge of the pions. Similar types of asymmetry have been observed in $p p \rightarrow \Lambda^{\uparrow} X{ }^{9]}$ and $\nu_{\mu} p \rightarrow$ $\mu \Lambda^{\uparrow} X \underline{10}$. It is expected that the underlying mechanisms of these different asymmetries are related, but it is also fair to say that single transverse spin asymmetries are not really understood, i.e., it is not yet clear how to explain them on the quark-gluon level. The suggested mechanisms can be roughly labeled as: semi-classical models; $\boldsymbol{k}_{T}$-dependent distributions; and, higher twist. Motivated by recent developments, the next section will mainly be about $\boldsymbol{k}_{T}$-dependent distributions.

First some short comments on the helicity dependence of transversity. A transverse spin state is an off-diagonal state in the helicity basis, which means that amplitudes with proton helicity + interfere with those of helicity -, see Fig. 3. For the transversity function $\delta q(x)$ the helicity flip of the proton states, is accompanied by helicity flip of the quark states, due to helicity conservation. In case one does not have helicity flip of the quark states, then one can satisfy helicity conservation by having an additional \pm 1 helicity gluon, at the cost of a suppression by one power of a large energy scale of the process. It is a twist-3 quark-gluon correlation inside a transversely polarized proton (Fig. 3). Neither $\delta q$, nor $g_{T}\left(=g_{1}+g_{2}\right)$ lead 


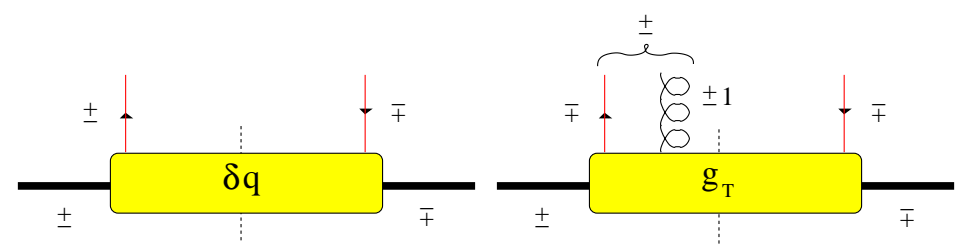

Figure 3. The helicity dependence of transversity $\delta q$ and the twist-3 function $g_{T}$.

to single transverse spin asymmetries in collinearly factorizing processes.

\section{Beyond collinear, leading twist factorization}

In order to describe single transverse spin asymmetries within a factorized approach, several ideas have been put forward, summarized in Fig. 4 Qiu
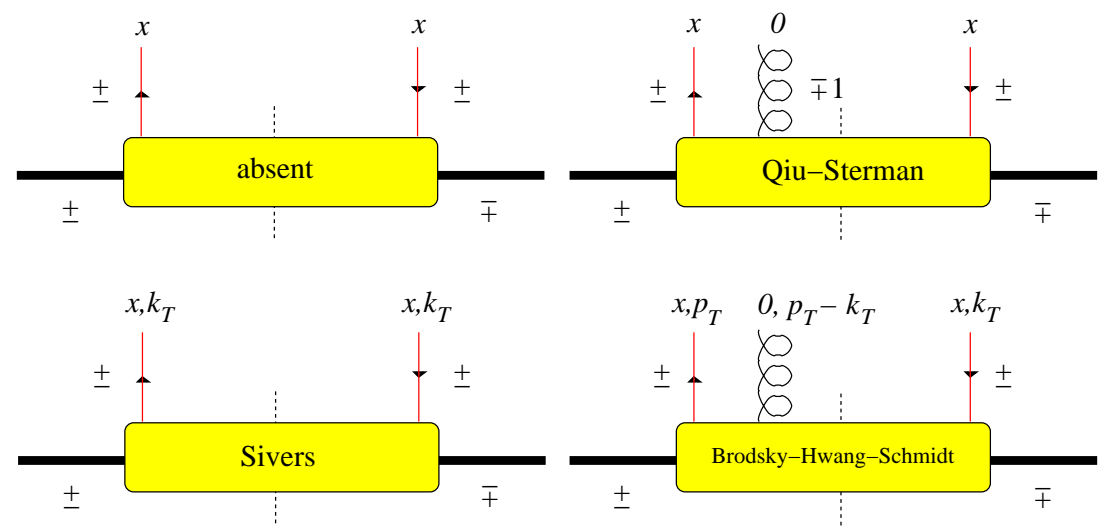

Figure 4. Pictorial representations of the Qiu-Sterman effect, the Sivers effect and the contribution considered by Brodsky, Hwang and Schmidt to generate nonzero SSA.

and Sterman ('91) 11 showed that the contribution where the gluon in the above-mentioned twist-3 quark-gluon correlation has vanishing momentum does give rise to a (suppressed) single spin asymmetry (SSA). Around the same time Sivers ('90) 12 suggested to consider quark momenta that are not completely collinear to the parent hadron's momentum. In that case one does not need helicity flip on the quark side to satisfy helicity conservation and an unsuppressed SSA could occur. However, Collins ('93) 13 demonstrated that this "Sivers effect" must be zero due to time reversal 
invariance. This demonstration turned out to be incorrect, as became clear after Brodsky, Hwang and Schmidt ('02) 14 obtained an unsuppressed SSA from a $\boldsymbol{k}_{T}$-dependent quark-gluon correlator (see Fig. 4) that is allowed by time reversal invariance. Belitsky, Ji and Yuan ('02) 15 showed that this particular correlator is a part of the proper gauge invariant definition of the Sivers function. After taking into account all numbers of gluons in this correlator, one obtains a path-ordered exponential in the off-lightcone, non-local operator matrix element that defines the Sivers function:

$$
f_{1 T}^{\perp} \propto\left\langle P, S_{T}\left|\bar{\psi}(0) \mathcal{L}[0, \xi] \gamma^{+} \psi(\xi)\right| P, S_{T}\right\rangle,
$$

where $\xi$ has (apart from an $n_{-}$component) a transverse component $\xi_{T}$. Collins ('02) 16 realized that the fact that the gauge invariant definition of the Sivers function in DIS contains a future pointing Wilson line (l.h.s. picture in Fig. 51), whereas in Drell-Yan (DY) it is past pointing (r.h.s. picture in Fig. (5), implies $\left(f_{1 T}^{\perp}\right)_{\text {DIS }}=-\left(f_{1 T}^{\perp}\right)_{\text {DY }}$. This calculable process
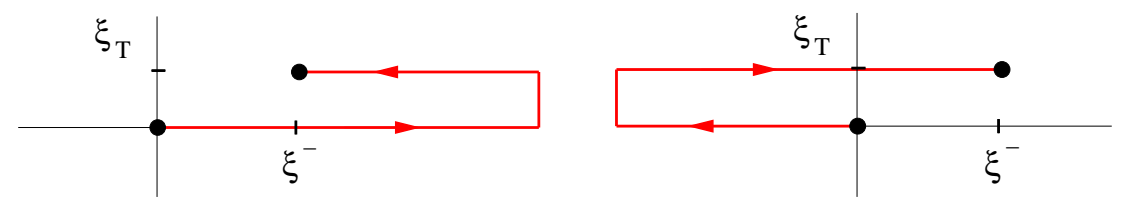

Figure 5. The links in DIS (l.h.s.) and DY run in opposite directions along the lightcone towards lightcone infinity, where an excursion in the transverse direction is taken.

dependence is an indication that the factorization is in terms of intrinsically nonlocal matrix elements, which are sensitive to certain aspects of the process as a whole. This does leave the still open question: what about more complicated processes?

After $\boldsymbol{k}_{T}$ integration both links reduce to the same link, namely the one we already encountered in $\Phi(x)$ (cf. Eq. (3)). On this latter quantity time reversal does pose the constraint that Collins initially derived for the Sivers function 13 , leading to the conclusion that no SSA can arise in fully inclusive DIS. This fact was already known at the level of structure functions: Christ and Lee ('66) 17 concluded that for the one-photon exchange approximation in inclusive DIS, only time-reversal violation can lead to a $\sin \phi_{S}^{e}$ SSA in $e p^{\uparrow} \rightarrow e^{\prime} X$.

Another way to represent the Sivers function and the three other leading $\boldsymbol{k}_{T}$-dependent (and often-called ' $T$-odd') functions is given in Figs. 60 where they are depicted as differences of probabilities. 


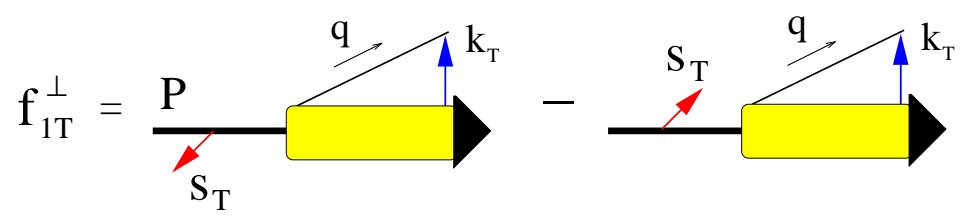

Figure 6. The Sivers effect distribution function. The proton $(P)$ is transversely polarized in direction $\boldsymbol{S}_{T}$ and the quark $(q)$ has a transverse momentum $\boldsymbol{k}_{T}$, such that the probability is proportional to $\boldsymbol{S}_{T} \times \boldsymbol{k}_{T}$.

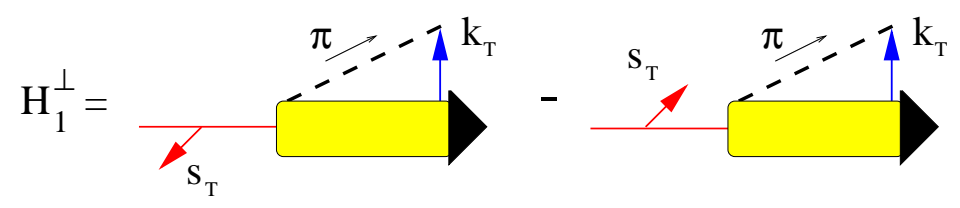

Figure 7. The Collins effect fragmentation function. Here the fragmenting quark is transversely polarized in direction $\boldsymbol{s}_{T}$ and the outgoing hadron (e.g. a pion) has $\boldsymbol{k}_{T}$.

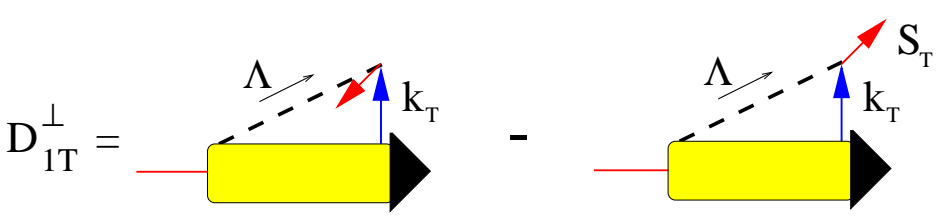

Figure 8. The fragmentation function $D_{1 T}^{\perp}$ 18. Now the outgoing, transversely polarized hadron (here a $\Lambda$ hyperon) has a transverse momentum $\boldsymbol{k}_{T}$.

\section{Azimuthal single spin asymmetries}

Apart from the left-right asymmetries, azimuthal spin asymmetries have been observed. In semi-inclusive DIS, $e p \rightarrow e^{\prime} \pi X$ (SIDIS), the HERMES Collaboration $\stackrel{20]}{ }$ has measured a nonzero $\sin \phi$ asymmetry in $e \vec{p}$ scattering $\left(A_{U L}\right)$ (for the definition of $\phi$ see Fig. 10). Also, preliminary data has been released (at this workshop) by HERMES on $e p^{\uparrow}$ scattering $\left(A_{U T}\right)$, suggesting that both Sivers and Collins effects are nonzero. In addition, the CLAS Collaboration (Jefferson Lab) has observed 21] a nonzero sin $\phi$ in $\vec{e} p$ scattering $\left(A_{L U}\right)$. These DIS data are at low $Q^{2}\left(\left\langle Q^{2}\right\rangle \sim 1-3 \mathrm{GeV}^{2}\right)$, so the interpretation of the asymmetries is not a straightforward matter. But they do demonstrate nontrivial spin effects, possibly related to the asymmetries of the $p p$ experiments. 


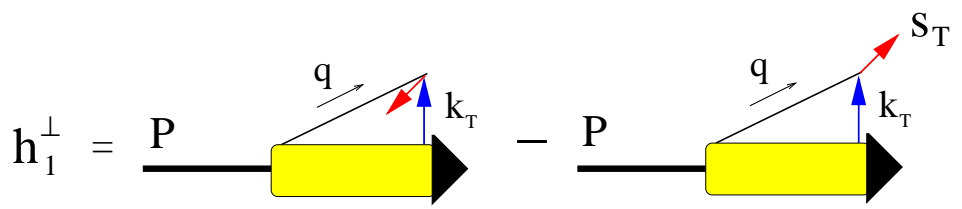

Figure 9. The distribution function $h_{1}^{\perp} 19$ describes transversely polarized quarks with nonzero transverse momentum inside an unpolarized hadron.

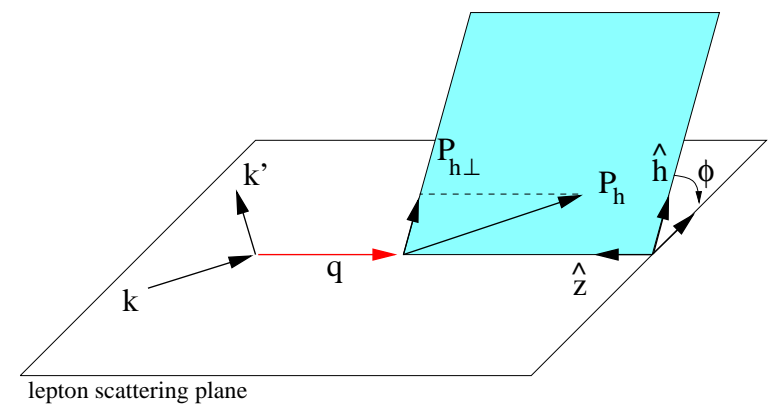

Figure 10. Kinematics of the semi-inclusive DIS process. The angle $\phi \equiv \phi_{\pi}^{e}$ is of the transverse momentum $\boldsymbol{P}_{h \perp}$ of the pion w.r.t. the lepton scattering plane, around the photon direction $\boldsymbol{q}$.

\subsection{Sivers and Qiu-Sterman effects}

The Sivers effect leads to a nonzero $A_{U T}$ (and also to $A_{U L}$, when the longitudinal spin is taken along the beam direction instead of the photon direction) and has the following characteristic angular dependence 19 .

$$
A_{U T} \propto \sin \left(\phi_{\pi}^{e}-\phi_{S}^{e}\right) f_{1 T}^{\perp[+]} D_{1},
$$

where the superscript $[+]$ indicates that a future pointing Wilson line appears in the Sivers function in this process. It is important to note that this Sivers effect asymmetry does not depend on the lepton scattering plane orientation, because at the parton level it arises from unpolarized quarkphoton scattering. Note also that there is no suppression by $1 / Q$ (except in $\left.A_{U L}\right)$.

Qiu and Sterman originally showed that the twist-3 matrix element

$$
\left.T_{F}^{(V)}(x, x)\right|_{A^{+}=0} \propto\left\langle\bar{\psi}(0) \Gamma_{\alpha} \int d \eta F^{+\alpha}\left(\eta n_{-}\right) \gamma^{+} \psi\left(\lambda n_{-}\right)\right\rangle
$$

can lead to a SSA in prompt photon production 11 ( $\Gamma_{\alpha}$ is an $S_{T}$-dependent 
Lorentz structure). But it can also lead to a nonzero $A_{U T}$ in SIDIS

$$
A_{U T} \propto \sin \left(\phi_{S}^{e}\right) \frac{T_{F}^{(V)}(x, x) D_{1}}{Q},
$$

where the expression applies after integrating over the transverse momentum of the pion. Note that this is not in conflict with the absence of a $\sin \left(\phi_{S}^{e}\right)$ asymmetry in inclusive DIS 17, where all final state hadrons are integrated out fully.

Recently, it was demonstrated that there is a direct relation between the Sivers and Qiu-Sterman effects 22 .

$$
f_{1 T}^{\perp(1)[+]}(x)=\frac{g}{2 M \vec{S}_{T}^{2}} T_{F}^{(V)}(x, x) .
$$

This expression contains a weighted Sivers function:

$$
f_{1 T}^{\perp(1)}(x) \equiv \int d^{2} \boldsymbol{p}_{T} \frac{\boldsymbol{p}_{T}^{2}}{2 M^{2}} f_{1 T}^{\perp}\left(x, \boldsymbol{p}_{T}^{2}\right) .
$$

So we conclude that the Sivers and Qiu-Sterman effects are not really different mechanisms after all.

\subsection{Collins effect}

The Collins effect is the only mechanism (within the formalism considered) that can lead to asymmetries $A_{U T}, A_{U L}$ and $A_{L U}$. For $A_{U T}$ it leads to

$$
A_{U T} \propto \sin \left(\phi_{S}^{e}+\phi_{\pi}^{e}\right)\left|\boldsymbol{S}_{T}\right| \delta q H_{1}^{\perp[-]},
$$

which does depend on the orientation w.r.t. the lepton scattering plane, because at the parton level it is transversely polarized quark scattering off the virtual photon. The asymmetry $A_{L U}$ from the Collins effect is $1 / Q$

suppressed 23, but can also be generated perturbatively at $\mathcal{O}\left(\alpha_{s}^{2}\right) 2425$ (only relevant if $\left|\boldsymbol{P}_{\perp}^{\pi}\right|^{2} \sim Q^{2}$, whereas here we consider $\left|\boldsymbol{P}_{\perp}^{\pi}\right|^{2} \ll Q^{2}$ ).

In Eq. (10) we have indicated the direction of the link in the definition of the Collins function in SIDIS. However, for fragmentation functions the implications of the link structure are not yet clear. On the basis of symmetry restrictions alone one finds schematically 22

$$
\left(H_{1}^{\perp}\right)_{\text {SIDIS }} \equiv A+B \quad \Rightarrow \quad\left(H_{1}^{\perp}\right)_{e^{+} e^{-}}=A-B .
$$

On the other hand, a model calculation by Metz $\frac{26}{26}$ shows that $B=0$. If this turns out to be true in general, it would simplify the comparison of Collins effect asymmetries from different processes. Clearly, this (calculable) process dependence must be studied further. 
Similar considerations apply to the process that may perhaps be of interest to the H1 and ZEUS experiments, namely $\stackrel{(\rightarrow)}{\ell} p \rightarrow \ell^{\prime} \Lambda^{\uparrow} X[27$,

$$
P_{N} \propto K_{1} \sin \left(\phi_{\Lambda}^{\ell}-\phi_{S}^{\ell}\right) f_{1} D_{1 T}^{\perp[-]}+K_{3} \sin \left(\phi_{\Lambda}^{\ell}+\phi_{S}^{\ell}\right) h_{1}^{\perp[+]} H_{1} .
$$

Here we would like to emphasize that all these asymmetry expressions apply to current fragmentation only.

\subsection{Scale dependence}

Sivers and Collins effect asymmetries are interesting observables, but are complicated from a theoretical viewpoint. The dependence on the hard scale $Q$ is highly non-trivial. Collinear factorization does not apply, since it is a multiscale process: $M,\left|\boldsymbol{P}_{\perp}^{\pi}\right|$ and $Q$ with $\left|\boldsymbol{P}_{\perp}^{\pi}\right|^{2} \ll Q^{2}$. If one considers the differential cross section for this not-fully-inclusive process, $d \sigma / d^{2} \boldsymbol{P}_{\perp}^{\pi}$, beyond tree level, then one finds that soft gluon corrections do not cancel, but rather exponentiate into Sudakov factors 28. These factors lead to a lowering and broadening (in transverse momentum) of the asymmetry with increasing $Q$. This decrease can be substantial, but one can define spe-

cific weighted asymmetries that are unaffected ${ }^{[29]}$ (apart from logarithmic corrections).

For the azimuthal spin asymmetries one finds 29 that in general, higher harmonics in the azimuthal angle $\phi_{\pi}^{e}$ decrease faster with $Q^{2}$. This is different from the azimuthal asymmetries generated perturbatively at higher orders in $\alpha_{s}$, where for instance the ratio $\langle\cos \phi\rangle /\langle\cos 2 \phi\rangle$ does not depend on Sudakov factors 30 .

\section{Conclusions}

Striking single spin asymmetries have been observed in experiment (leftright asymmetries and $\sin \phi$ azimuthal asymmetries), but these are still not understood. By using collinear factorization at leading twist, one will not be able to describe these asymmetries, even if one includes higher order perturbative QCD corrections.

Some insights about possible mechanisms for single spin asymmetries are that: the Sivers effect is allowed by time reversal invariance; in SIDIS and Drell-Yan it is opposite in sign; $\boldsymbol{k}_{T}$-dependent functions may lead to unsuppressed asymmetries; and, the Qiu-Sterman and Sivers effects are directly related. Issues that require further study are: the calculable process dependence of $\boldsymbol{k}_{T}$-dependent functions (especially of fragmentation func- 
tions); the possible connection between the Sivers effect and orbital angular momentum 31, and, the $Q^{2}$ dependence of azimuthal spin asymmetries.

\section{Acknowledgments}

I thank the organizers of this interesting workshop for their kind invitation. Some results presented here were obtained in collaboration with Piet Mulders and Fetze Pijlman. The research of D.B. has been made possi-

ble by financial support from the Royal Netherlands Academy of Arts and Sciences.

\section{References}

1. A.V. Efremov and A.V. Radyushkin, Theor. Math. Phys. 44, 774 (1981).

2. X. Ji, Phys. Rev. D49, 114 (1994).

3. J.C. Collins, S.F. Heppelmann, G.A. Ladinsky, Nucl. Phys. B420, 565 (1994).

4. R.L. Jaffe, X. Jin and J. Tang, Phys. Rev. Lett. 80, 1166 (1998).

5. A. Bianconi et al., Phys. Rev. D62, 034008 (2000).

6. FNAL E704 Collaboration, D.L. Adams et al., Phys. Lett. B261, 201 (1991).

7. K. Krueger et al., Phys. Lett. B459, 412 (1999).

8. STAR Collaboration, J. Adams et al., hep-ex/0310058

9. G. Bunce et al., Phys. Rev. Lett. 36, 1113 (1976).

10. NOMAD Collaboration, P. Astier et al., Nucl. Phys. B588, 3 (2000).

11. J. Qiu and G. Sterman, Phys. Rev. Lett. 67, 2264 (1991).

12. D. Sivers, Phys. Rev. D41, 83 (1990); Phys. Rev. D43, 261 (1991).

13. J.C. Collins, Nucl. Phys. B396, 161 (1993).

14. S.J. Brodsky, D.S. Hwang and I. Schmidt, Phys. Lett. B530, 99 (2002).

15. A.V. Belitsky, X. Ji and F. Yuan, Nucl. Phys. B656, 165 (2003).

16. J.C. Collins, Phys. Lett. B536, 43 (2002).

17. N. Christ and T.D. Lee, Phys. Rev. 143, 1310 (1966).

18. P.J. Mulders and R.D. Tangerman, Nucl. Phys. B461, 197 (1996).

19. D. Boer and P.J. Mulders, Phys. Rev. D57, 5780 (1998).

20. HERMES Collab., A. Airapetian et al., Phys. Rev. Lett. 84, 4047 (2000).

21. CLAS Collaboration, H. Avakian et al., hep-ex/0301005

22. D. Boer, P.J. Mulders and F. Pijlman, Nucl. Phys. B667, 201 (2003).

23. J. Levelt and P.J. Mulders, Phys. Lett. B338, 357 (1994).

24. K. Hagiwara, K. Hikasa and N. Kai, Phys. Rev. D27, 84 (1983).

25. M. Ahmed and T. Gehrmann, Phys. Lett. B465, 297 (1999).

26. A. Metz, Phys. Lett. B549, 139 (2002).

27. D. Boer, R. Jakob and P.J. Mulders, Nucl. Phys. B564, 471 (2000).

28. J.C. Collins and D. Soper, Nucl. Phys. B193, 381 (1981).

29. D. Boer, Nucl. Phys. B603, 195 (2001).

30. P. Nadolsky, D.R. Stump and C.-P. Yuan, Phys. Lett. B515, 175 (2001).

31. M. Burkardt and D.S. Hwang, hep-ph/0309072 